\title{
m-AAA proteases, mitochondrial calcium homeostasis and neurodegeneration
}

\author{
Maria Patron $^{1,2}$, Hans-Georg Sprenger ${ }^{1,2}$, Thomas Langer ${ }^{1,2}$ \\ ${ }^{1}$ Max Planck Institute for Biology of Aging, Cologne, Germany; ${ }^{2}$ Cologne Excellence Cluster on Cellular Stress Response in Ag- \\ ing-Associated Disease (CECAD), and Center for Molecular Medicine Cologne (CMMC), University of Cologne, Cologne, Ger- \\ many
}

The function of mitochondria depends on ubiquitously expressed and evolutionary conserved $\boldsymbol{m}$-AAA proteases in the inner membrane. These ATP-dependent peptidases form hexameric complexes built up of homologous subunits. AFG3L2 subunits assemble either into homo-oligomeric isoenzymes or with SPG7 (paraplegin) subunits into hetero-oligomeric proteolytic complexes. Mutations in $A F G 3 L 2$ are associated with dominant spinocerebellar ataxia (SCA28) characterized by the loss of Purkinje cells, whereas mutations in SPG7 cause a recessive form of hereditary spastic paraplegia (HSP7) with motor neurons of the cortico-spinal tract being predominantly affected. Pleiotropic functions have been assigned to $m$-AAA proteases, which act as quality control and regulatory enzymes in mitochondria. Loss of $m$-AAA proteases affects mitochondrial protein synthesis and respiration and leads to mitochondrial fragmentation and deficiencies in the axonal transport of mitochondria. Moreover $m$-AAA proteases regulate the assembly of the mitochondrial calcium uniporter (MCU) complex. Impaired degradation of the MCU subunit EMRE in AFG3L2-deficient mitochondria results in the formation of deregulated MCU complexes, increased mitochondrial calcium uptake and increased vulnerability of neurons for calcium-induced cell death. A reduction of calcium influx into the cytosol of Purkinje cells rescues ataxia in an AFG3L2-deficient mouse model. In this review, we discuss the relationship between the $\boldsymbol{m}$-AAA protease and mitochondrial calcium homeostasis and its relevance for neurodegeneration and describe a novel mouse model lacking MCU specifically in Purkinje cells. Our results pledge for a novel view on $m$-AAA proteases that integrates their pleiotropic functions in mitochondria to explain the pathogenesis of associated neurodegenerative disorders.

Keywords: mitochondria; m-AAA proteases; AFG3L2; SPG7; spinocerebellar ataxia; hereditary spastic paraplegia; mitochondrial calcium uniporter

Cell Research (2018) 28:296-306. doi:10.1038/cr.2018.17; published online 16 February 2018

\section{Introduction}

Mitochondria are dynamic, double-membrane-bound organelles that reside in all eukaryotic cells with a prominent role in metabolism, ion homeostasis and cell death.

Correspondence: Thomas Langer

Tel: +49 22137970 500; Fax: +49 2213797088500

E-mail: Thomas.Langer@age.mpg.de

Abbreviations: AMPA, a-amino-3-hydroxyl-5-methyl-4-isoxazole-propionate; CNS, central nervous system; DCN, deep cerebellar nuclei; GCL, granule cells layer; HSP, hereditary spastic paraplegia; IMM, inner mitochondrial membrane; IMS, intra membrane space; mGluR, metabotropic glutamate receptors; ML, molecular cell layer; PCL, Purkinje cell layer; PNS, peripheral nervous system; SCA, spinocerebellar ataxia; SPAX, spastic ataxia
They exert essential anabolic and catabolic functions and produce ATP through oxidative phosphorylation, while mitochondrial outer membrane permeabilization, regulated by Bcl-2 family proteins, induces the intrinsic apoptotic pathway. ATP synthesis depends on the maintenance of a proton gradient across the mitochondrial inner membrane, which exhibits low permeability to ions. Still, the membrane harbors a variety of highly regulated, selective and non-selective ion channels, which preserve mitochondrial and cellular ion homeostasis.

Perturbations of mitochondrial functions are associated with ageing and the development of many pathological conditions, highlighting the central role of mitochondria for cell survival and tissue homeostasis. Numerous pathogenic mutations in nuclear-encoded mitochondrial 
proteins have been identified that often cause neuromuscular disorders. This is exemplified by proteases, which reside in different mitochondrial compartments and preserve the functional integrity of mitochondria $[1,2]$. They constitute an intra-organellar quality control system, which ensures the complete degradation of misfolded and damaged mitochondrial proteins to amino acids. Moreover, multiple processes in mitochondria are under the control of proteases, which mediate processing events and limit the stability of short-lived mitochondrial proteins (Figure 1). Thereby, proteases regulate the import of nuclear-encoded proteins into mitochondria, the stability of the mitochondrial genome and the synthesis of mitochondrially encoded subunits of the respiratory chain complexes. Similarly, the synthesis and intramitochondrial transport of phospholipids and the dynamic behavior of mitochondrial membranes that constantly fuse and divide are under proteolytic control. Considering the pleiotropic roles of proteases for the functional integrity of mitochondria, it is not surprising that a steadily increasing number of pathogenic mutations are being identified in genes encoding mitochondrial proteases (Table 1). However, the pathogenic mechanisms underlying the resulting diseases may vary significantly and to account for them, the contributions of the loss of regulatory functions in combination with an impaired mitochondrial quality control must be considered.

\section{AAA proteases - proteolytic machines in the mito- chondrial inner membrane}

AAA proteases constitute a family of conserved and ubiquitous ATP-dependent proteases that are embedded in the mitochondrial inner membrane. Subunits of these hexameric proteolytic complexes harbor an ATPase domain of the AAA family (ATPases associated with diverse cellular activities) and a metallopeptidase of the M48 family. AAA proteases exposing these catalytic domains to the matrix are termed $m$-AAA proteases in contrast to the $i$-AAA proteases, which exhibit an inverted topology in the inner membrane with catalytic domains protruding into the intermembrane space. An $i$-AAA protease is composed of YME1L subunits whereas various isoenzymes of mammalian $m$-AAA proteases have homologous subunits but differ in their subunit composition [3-5]. For example, AFG3L2 subunits can either form homo-oligomeric complexes or hetero-oligomerize with SPG7 (paraplegin) subunits that only assemble into hetero-oligomeric $m$-AAA proteases. Moreover, an additional $m$-AAA protease subunit, AFG3L1, is expressed in rodents [6] and can either homo-oligomerize or assemble with AFG3L2 and SPG7 subunits [4].

Studies on yeast homologues of $m$ - and $i$-AAA proteases provided the first insight into the structural organization of mitochondrial AAA proteases [7] and revealed principles of membrane protein degradation by these proteolytic machines [8-12]. The assembly of AAA proteases into hexameric ring complexes allows proteolysis to occur in a protected environment within a proteolytic chamber. The chaperone-like activity of AAA domains ensures the specific recognition of misfolded,

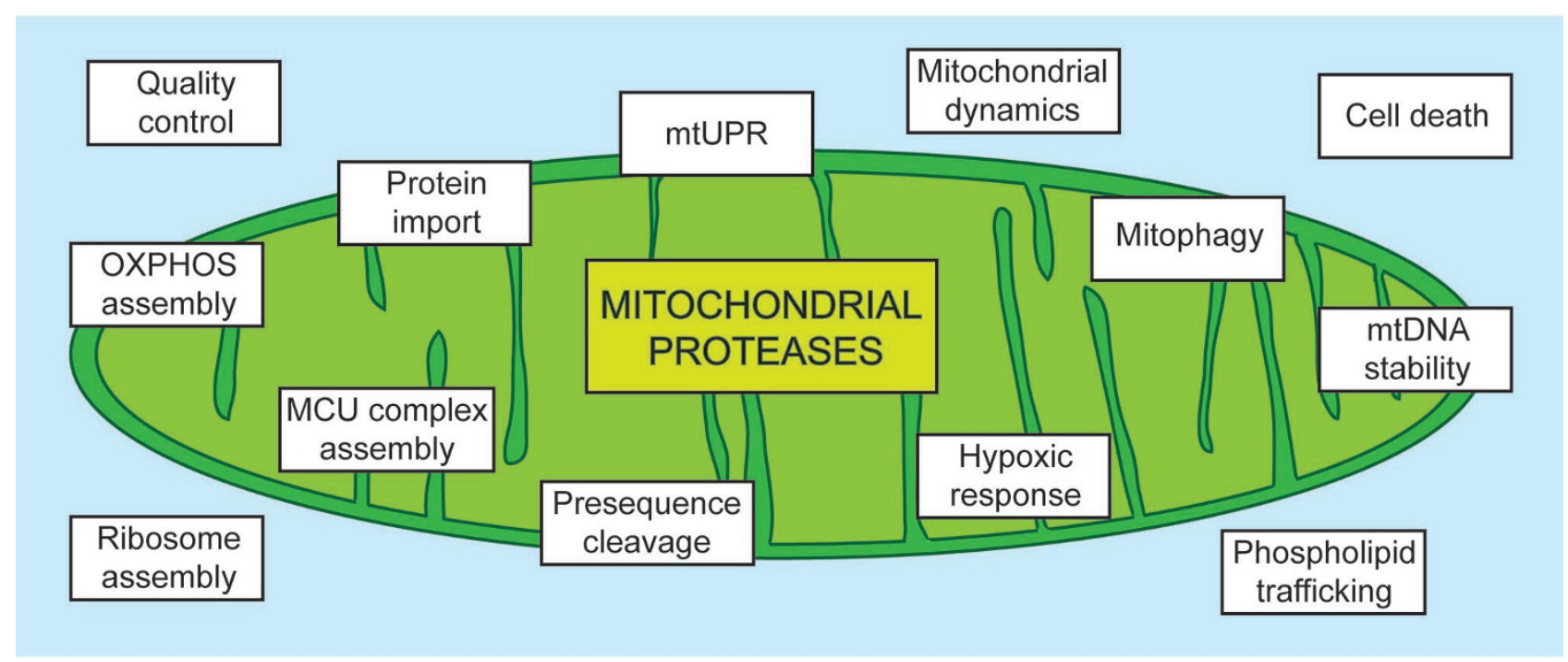

Figure 1 Functions of mitochondrial proteases. Mitochondrial proteases execute multiple functions that are crucial for mitochondrial function. Besides protein quality surveillance, many proteases control a variety of processes within mitochondria (shown in boxes) by either proteolytic processing or by mediating the rapid turnover of regulatory proteins. 
Table 1 Diseases associated with loss of protease function

\begin{tabular}{|c|c|c|c|c|}
\hline Gene & Disease & Inheritance & OMIM & References \\
\hline$A F G 3 L 2$ & $\begin{array}{l}\text { Spinocerebellar ataxia type } 28 \text { (SCA28); } \\
\text { spastic ataxia-neuropathy syndrome }\end{array}$ & Dominant; recessive & $610246 ; 614487$ & {$[17,19]$} \\
\hline HTRA2 & Parkinson disease type 13 & Dominant & 610297 & {$[70-72]$} \\
\hline$I M M P 2 L$ & Gilles de la Tourette syndrome & Dominant & 137580 & [73] \\
\hline$P M P C B$ & Friedreich ataxia & Recessive & 229300 & {$[76,77]$} \\
\hline$S P G 7$ & Hereditary spastic paraplegia 7 (HSP7) & Recessive & 607259 & {$[16,35]$} \\
\hline$X P N P E P 3$ & Nephronophthisis-like nephropathy 1 & Recessive & 613159 & {$[78]$} \\
\hline$Y M E 1 L$ & Optic atrophy 11 & Recessive & 617302 & {$[15]$} \\
\hline
\end{tabular}

OMIM, Online mendelian inheritance in Man database; AFG3L2, AFG3-like protein 2; CLPP, ATP-dependent Clp protease proteolytic subunit; HTRA2, high-temperature requirement protein A2; IMMP2L, mitochondrial inner membrane protease 2; LONP1, lon protease homologue; PARL, presenilins-associated rhomboidlike protein; PMPCB, peptidase mitochondrial processing beta subunit; SPG7, paraplegin; XPNPEP3, X-Pro aminopeptidase 3; YME1L1, ATP-dependent zinc metalloprotease YME1L1.

solvent exposed domains of membrane proteins $[8,9]$. After binding to the outer surface of AAA proteases, substrate proteins are extracted from the membrane bilayer into the proteolytic chamber for degradation $[9,10,13]$. Substrate dislocation is mediated by coordinated ATP hydrolysis with the ring of ATPase domains and central pore loops directly binding to substrate proteins $[10,12]$. AAA proteases exhibit a degenerate substrate specificity and can mediate the complete degradation of proteins to peptides, but structural constraints in substrate proteins, such as tightly folded domains, may limit proteolysis and allow proteolytic processing [14]. This functional plasticity makes AAA proteases versatile proteolytic machines with multiple functions within mitochondria.

\section{AAA proteases and neurodegeneration}

Mutations in genes encoding AAA protease subunits have been associated with neuronal loss and neurodegeneration in humans. Homozygous mutations in YME1L were found to cause a mitochondriopathy with optic atrophy in a consanguineous pedigree [15] but it remains to be seen whether mutations in $Y M E 1 L$ are of broader pathogenic relevance. Recessive mutations in $S P G 7$ coding for the $m$-AAA protease subunit paraplegin cause hereditary spastic paraplegia (HSP7) [16]. The main clinical features of this frequent mitochondriophathy are weakness and spasticity of the lower limbs, loss of vibratory sense and urinary urgency due to degeneration of motor axons of the cortico-spinal tracts. Manifestations of the disease can also include cortical and cerebellar atrophy, amyotrophy and mental retardation. Mutations in $A F G 3 L 2$ coding for the second $m$-AAA protease subunit are associated with spinocerebellar ataxia type 28 (SCA28), which is accompanied by the loss of Purkinje cells [17]. SCA28 is an autosomal dominantly inherited, rare ataxia with juvenile-onset, which is characterized by progressive gait and limb ataxia with eye movement abnormalities due to cerebellar abnormalities [18].

The association of mutations in $S P G 7$ and $A F G 3 L 2$ with distinct neurodegenerative disorders can be explained by the formation of different isoenzymes by the two subunits: whereas the loss of SPG7 impairs specifically the formation of hetero-oligomeric m-AAA proteases, both homo- and hetero-oligomeric forms are affected in the absence of AFG3L2. As both isoenzymes appear to exert largely overlapping substrate specificities, the relative expression of $S P G 7$ and $A F G 3 L 2$ may contribute to the cell-type specificity in disease. This interpretation is consistent with the identification of recessive mutations in $A F G 3 L 2$ that affect the interaction of AFG3L2 with SPG7 as causative for spastic ataxia 5 (SPAX5). SPAX5 is a severe, early onset autosomal recessive ataxia, which is characterized by impaired ambulation, cerebellar ataxia and dystonia, thus combining clinical features of both HSP7 and SCA28 [19].

\section{Studying neuronal loss in $\boldsymbol{m}$-AAA protease-defi- cient mouse models}

The expression of $\operatorname{Spg} 7$ and $A f g 3 l 2$ varies significantly between different tissues in mice, with a tenfold higher 
expression of $A f g 3 l 2$ and a fourfold higher expression of Spg7 in the brain when compared to liver mitochondria [4]. Moreover, differences exist between the relative expression of $S p g 7$ and $A f g 3 l 2$ in the mouse brain, suggesting a prominent role for AFG3L2 in the brain and cooperative role for SPG7 in hippocampal neurons and Purkinje cells [20]. Different expression levels of Spg7 and Afg $3 l 2$ and the presence of homo- and hetero-oligomeric $m$-AAA proteases in different amounts may therefore contribute to the cell-type specificity in disease. Notably, the rodent-specific $m$-AAA protease subunit AFG3L1 is only expressed at low levels in the mouse brain. The loss of SPG7 or AFG3L2 in neurons therefore mimics the situation in humans, making mice a suitable model system to study the pathogenic mechanism of HSP7 and SCA28.

Mice lacking $\operatorname{Spg} 7$ show mild and slowly progressive motor impairment associated with distal axonopathy of spinal and peripheral axons [21]. Mitochondrial morphological abnormalities occur in synaptic terminals and in distal regions of axons before axonal swelling and onset of motor impairment [21]. On the other hand, homozygous missense mutations in $\mathrm{Afg} 3 \mathrm{l2}$ in a spontaneous mutant strain and the loss of AFG3L2 in $A f g 3 l 2^{-/}$mice cause a severe neuromuscular phenotype with hind limbs paresis, a severe defect in axonal development with delayed myelination and impairment of axonal radial growth, culminating in death shortly after birth. Mitochondrial morphology in these mice is abnormal with a strong impairment of the respiratory chain complexes resulting in reduced mitochondrial ATP production [22]. Thus, as in human disease, mutations in $\mathrm{Spg} 7$ and $\mathrm{Afg} 3 \mathrm{l} 2$ cause distinct phenotypes in mice. Afg 312 -mutated mice show alterations in both the central nervous system (CNS) and peripheral nervous system with the cerebellum being the most strongly affected. The severe consequences of the loss of AFG3L2 can be explained by the inability of SPG7 to form m-AAA protease complexes in the absence of AFG3L2, whereas AFG3L2 is able to assemble into functional, homo-oligomeric complexes when SPG7 is absent. Notably, despite similar expression levels of Spg7 and Afg $3 l 2$ in murine astrocytes, oligodendrocytes and neurons, the latter are more susceptible to mutations in $A f g 3 l 2$, likely reflecting differences in the metabolic profiles of these cell types [23].

To delineate pathogenic mechanisms underlying neurodegeneration in AFG3L2 deficiency, functional studies have been performed in heterozygous $A f g 3 l 2^{+/-}$mice. These are largely normal and do not show impaired axonal development but display a mild late-onset degenerative phenotype at least in certain experimental settings [24]. The progressive deficit in motor coordination in Afg $3 l 2^{+-}$mice is the result of dark cell degeneration of
Purkinje cells, characterized morphologically by cytoplasmic darkening, nuclear condensation and neuronal shrinkage. In these cells mitochondria appear swollen with disrupted cristae [24] and show an impaired ability for trafficking and buffering cytosolic calcium $\left(\mathrm{Ca}^{2+}\right)$ waves [25]. Neurological deficiencies are aggrevated when $\mathrm{Afg} 3 \mathrm{l} 2^{+/-}$mice are bred with $\mathrm{Spg} 7^{-/-}$mice [20]. Such mice show loss of balance, tremor and ataxia, characterized by reactive astrogliosis and prominent cerebellar degeneration with loss of Purkinje cells and parallel fibers [20]. These phenotypes highlight the critical role of the expression levels of $m$-AAA protease subunits for neuronal survival.

\section{Delineating the pathogenic mechanisms of neuro- degeneration in SCA28}

Purkinje cells exclusively receive excitatory synapsis and, as the only efferent projections from the cortex, they modulate the activity of others neurons in the deep cerebellar nuclei (DCN). Accordingly, loss of these cells leads to hyper-excitable neurons of DCN with immediate consequence on the regulation of the fine movement [26]. Purkinje cells express high levels of AFG3L2 and SPG7 and are highly vulnerable towards the loss of the $m$-AAA protease in SCA28. Deletion of $A f g 3 l 2$ specifically in Purkinje cells triggers mitochondrial fragmentation and an altered distribution of mitochondria in the dendritic tree [27]. Accordingly, AFG3L2 is required for anterograde axonal transport of mitochondria in murine cortical neurons, suggesting that impaired mitochondrial trafficking leads to the progressive depletion of mitochondria from axons causing the late onset of the disease [28].

Further studies in cultured cells have unraveled the mechanism of how the $m$-AAA protease influences mitochondrial morphology. Loss of the $m$-AAA protease activates the inner membrane peptidase OMA1 [29], which specifically cleaves the dynamin-like GTPase OPA1, a central component of the mitochondrial fusion machinery [30]. OMA1 limits the accumulation of fusion-active long OPA1 variants and leads to mitochondrial fragmentation due to ongoing fission events [29-32]. Misfolded, mitochondrial-encoded proteins accumulating in the absence of the $m$-AAA protease were found to activate OMA1 and to induce mitochondrial fragmentation [29, $33,34]$. The $m$-AAA protease is thus emerging as an important sensor coordinating the synthesis from mitochondrial and nuclear genomes. However, OMA1 activation upon loss of AFG3L2 and mitochondrial fragmentation apparently does not play a prominent role in axonal degeneration, as ablation of Oma1 in AFG3L2-deficient neurons restores mitochondrial tubulation but not the ax- 
onal transport of mitochondria [28, 29]. Thus, mitochondrial fragmentation appears to occur secondarily to other mitochondrial deficiencies.

AFG3L2-deficient Purkinje cells show respiratory defects and at early stages accumulate mitochondrial-encoded respiratory chain subunits at reduced levels [27], defects that could explain the fragmentation of the mitochondrial network. The $m$-AAA protease is required for the synthesis of mitochondrial-encoded proteins in liver and brain mitochondria $[27,35]$ and regulates the assembly of mitochondrial ribosomes [27]. Studies in yeast have revealed that the $m$-AAA protease mediates the proteolytic processing of the ribosomal protein MrpL32, a prerequisite for mitochondrial translation $[14,35]$. While it remains to be demonstrated how the $m$-AAA protease regulates ribosome assembly and gene expression in mammalian mitochondria, it is conceivable that defective mitochondrial protein synthesis and respiratory defects are central pathogenic factors in AFG3L2-related neurodegeneration [27]. In agreement with this hypothesis, antioxidants or decreasing phospho-tau levels were found to rescue axonal trafficking defects in neurons lacking AFG3L2 [28], suggesting that reactive oxygen species signaling leads to cytoskeletal modifications that impair the axonal transport of mitochondria.

\section{$\mathrm{Ca}^{2+}$-dependent neuronal death and neurological diseases}

These studies unraveled fundamental roles of $m$-AAA proteases for the functional integrity of mitochondria and neuronal survival, but the striking cell-type specificity of related neurodegenerative disorders remains unexplained. What are the features of the target cells that make them especially vulnerable to the loss of AFG3L2 or SPG7? Purkinje cells that are predominantly affected in ataxias are characterized by high-firing frequencies and express a variety of $\mathrm{Ca}^{2+}$ channels, $\mathrm{Ca}^{2+}$-sensitive phosphatases and kinases, and $\mathrm{Ca}^{2+}$-binding proteins to ensure efficient $\mathrm{Ca}^{2+}$ buffering [36]. Indeed, increasing evidence suggests that impaired cellular $\mathrm{Ca}^{2+}$ signaling plays a role in the etiology of various spinocerebellar ataxias [36, 37] (Table 2). Mitochondria can take up $\mathrm{Ca}^{2+}$ ions released from the endoplasmic reticulum, the major cellular $\mathrm{Ca}^{2+}$ store [38]. Being localized in close proximity to the plasma membrane, they also modulate extracellular $\mathrm{Ca}^{2+}$ influx, a mechanism that is specifically important in neurons [39]. They thus can act as a local buffer system determining the rate of $\mathrm{Ca}^{2+}$ waves through the cytosol. $\mathrm{Ca}^{2+}$ sequestration by mitochondria in the synapse can shape dynamic alterations in the cytosolic $\mathrm{Ca}^{2+}$ levels and strongly affect neurotransmitter release. Local alterations in the cytosolic $\mathrm{Ca}^{2+}$ concentration can position mitochondria to specific neuronal areas and the recruitment of mitochondria to specific areas changes the ion balance by controlling $\mathrm{Ca}^{2+}$ influx or release [40].

Massive accumulation of $\mathrm{Ca}^{2+}$ ions in the matrix increases the permeability of the IMM by opening the mitochondria permeability transition pore (mPTP) [41]. Prolonged mPTP opening causes the collapse of the proton gradient, inhibition of ATP synthesis and dissipation of the ionic gradient across the IMM, which causes swelling, cristae unfolding and OMM rupture [42]. This mechanism also favors the release of pro-apoptotic proteins like cytochrome c. Deregulated $\mathrm{Ca}^{2+}$ homeostasis and MPTP opening are associated with many diseases of the CNS suggesting beneficial effects of an inhibition of the permeability transition mechanism [43].

The first evidence for an important role of impaired $\mathrm{Ca}^{2+}$ homeostasis in SCA28 came from recent studies on heterozygous $\mathrm{Afg} 3 \mathrm{l2}^{+/-}$mice demonstrating that reducing the $\mathrm{Ca}^{2+}$ influx through the plasma membrane ameliorates motor deficits and dark cell degeneration of Purkinje cells [25]. In Purkinje cells, a transient increase in the cy-

Table 2 Role of $\mathrm{Ca}^{2+}$ in the etiology of SCA

\begin{tabular}{llll}
\hline Type of SCA & Protein & Effect on $\mathbf{C a}^{2+}$ homeostasis & References \\
\hline SCA1 & Ataxin 1 & Reduction of $\mathrm{PC} \mathrm{Ca}^{2+}$-binding protein levels & {$[79,80]$} \\
SCA2 & Ataxin 2 & Increase $\mathrm{Ca}^{2+}$ release from ER stores & {$[81,82]$} \\
SCA3 & Ataxin 3 & Increase $\mathrm{Ca}^{2+}$ release from ER stores \\
SCA5 & $\beta$-III-spectrin & Increase & {$[83]$} \\
SCA6 & CACNA1A & Impaired Ca ${ }^{2+}$ flux into neurons \\
SCA14 & PKC $\gamma$ & Increase/Decrease & {$[85]$} \\
SCA15 & ITPR1 & Inositol 1,4,5-triphosphate Ca ${ }^{2+}$ signaling \\
SCA28 & AFG3-like protein 2 & MCU complex assembly & {$[86]$} \\
\hline CACNA
\end{tabular}

CACNA1A, $\mathrm{Ca}^{2+}$ voltage-gated channel subunit alpha1 A; PKC $\gamma$, protein kinase $\mathrm{C}$ subunit $\gamma$; ITPR1, inositol 1,4,5-trisphosphate receptor type 1; SCA, spinocerebellar ataxias. 
tosolic $\mathrm{Ca}^{2+}$ level can be induced by glutamate via a-amino-3-hydroxyl-5-methyl-4-isoxazole-propionate (AMPA) receptors or by the release of $\mathrm{Ca}^{2+}$ from the endoplasmic reticulum via metabotropic glutamate receptors (mGluR). Strikingly, reducing the dosage of mGluR1 by one half restores the motor performance of $A f g 3 l 2^{+/-}$mice [25]. Similarly, when $\mathrm{Afg} 3 \mathrm{l} 2^{+/-}$mice are fed with the antibiotic ceftriaxone promoting the clearance of glutamate from synapses, this suppresses the ataxic phenotype [45], further substantiating the beneficial effects of reducing the cytosolic $\mathrm{Ca}^{2+}$ concentration in AFG3L2-deficient Purkinje cells.

These studies point to a prominent role of disturbed cellular $\mathrm{Ca}^{2+}$ homeostasis in the pathogenesis of SCA28. It has been suggested that mitochondrial fragmentation [46] or mitochondrial depolarization [25] impairs $\mathrm{Ca}^{2+}$ influx into AFG3L2-deficient mitochondria resulting in an increase of cytosolic $\mathrm{Ca}^{2+}$ concentrations and neuronal death. However, as mitochondrial $\mathrm{Ca}^{2+}$ buffering affects mainly local $\mathrm{Ca}^{2+}$ concentrations in the cytosol, alternative scenarios explaining the disturbed $\mathrm{Ca}^{2+}$ homeostasis in AFG3L2-deficient neurons must also be considered. Notably, recent work by Shanmughapriya et al. identified SPG7 amongst the proteins that co-immunoprecipitate with the well-known PTP regulator CyPD and suggested SPG7 to be an essential component of the mitochondrial PTP complex together with VDAC1 [44]. Although such a function is difficult to reconcile with neuronal loss in HSP patients lacking SPG7, a possible regulatory function of SPG7 in the formation of PTP warrants further investigation.

\section{The neuronal interactome of $\boldsymbol{m}$-AAA proteases}

The striking cell-type specificity of $m$-AAA protease related diseases may reflect an increased dependence of Purkinje cells on AFG3L2 or the impaired proteolysis of neuron-specific substrates. The recent determination of the neuronal interactome of $m$-AAA proteases in mice provided the first insight into this question [47]. A point mutation in the Walker B motif of the ATPase domain of AFG3L2 inactivates the $m$-AAA protease but does not interfere with its assembly or with the binding of substrate proteins $[12,29]$. Importantly, this variant exerts a dominant negative effect on the activity of endogenous $m$-AAA proteases [29] and its expression in the mouse forebrain therefore phenocopies the loss of AFG3L2 in vivo inducing atrophy and neuronal death [47]. Affinity purification of mutant $m$-AAA proteases from mouse brain led to the identification of the $\underline{m}$-AAA protease-interacting protein 1 (MAIP1). MAIP1 is a ubiquitously expressed protein that, rather than being degraded, stably associates with $m$-AAA protease complexes. It contains a TIMM44-like domain, which is suggested to exert chaperone-like activity and which is also found in the mitochondrial protein translocase subunit TIMM44 and the mitochondrial ribosomal subunit MRPL45 (Mba1 in yeast). Interestingly, the $\alpha$-proteobacterial AAA protease FtsH forms a complex with a related protein containing a TIMM44-like domain indicating that this complex may be an evolutionary ancestor of mitochondrial protein translocases [48]. MAIP1 is not required for the proteolytic activity of the $m$-AAA protease but it regulates the membrane insertion of the single span inner membrane protein EMRE, an essential subunit of the mitochondrial $\mathrm{Ca}^{2+}$ uniporter (MCU) complex [49], linking the function of the $m$-AAA protease to mitochondrial ion homeostasis [47]. Further studies identified EMRE as a novel substrate of the $m$-AAA protease, which degrades non-assembled EMRE subunits and thereby ensures MCU assembly and mitochondrial $\mathrm{Ca}^{2+}$ homeostasis $[47,50]$.

\section{The m-AAA protease regulates MCU assembly and mitochondrial $\mathrm{Ca}^{2+}$ homeostasis}

The MCU complex allows the regulated transport of $\mathrm{Ca}^{2+}$ ions across the inner membrane driven by the electrical gradient [51-53]. It consists of a pore-forming unit that includes MCU itself, a dominant negative variant MCUb [54], a small protein EMRE [49], and the MICU family that functionally shape the channel activity [5559]. At resting conditions, MICU proteins act as a lid and prevent uncontrolled $\mathrm{Ca}^{2+}$ influx into the mitochondrial matrix, whereas they serve as positive activators when the $\mathrm{Ca}^{2+}$ concentration overcomes a specific threshold. This mechanism of regulation allows $\mathrm{Ca}^{2+}$ to fuel the Krebs cycle without inducing cell death. EMRE bridges MCU subunits with the C-terminal poly-aspartate tail of MICU1 (the main component of the MICU family) [57]. It is required for MCU channel activity [49, 60] but at the same time keeps the regulatory proteins of the MICU family bound to the pore-forming unit [61]. The analysis of MCU assembly revealed that EMRE, present at low levels, interacts with regulatory proteins of the MICU family before it assembles with MCU subunits into a functionally active MCU complex, illustrating the importance of a tight control of mitochondrial $\mathrm{Ca}^{2+}$ influx during MCU assembly [47]. Loss of MICU1 or disruption of the EMRE-MICU interaction leads to the dissociation of the regulatory proteins from the MCU complex, resulting in the accumulation of MCU-EMRE complexes that are constitutively open and allow accumulation of $\mathrm{Ca}^{2+}$ ions in the matrix [61-63].

A similar effect on MCU assembly has been observed 
in $m$-AAA protease-deficient neurons. Degradation of non-assembled EMRE by the $m$-AAA protease ensures the binding of EMRE-MICU assembly intermediates with MCU subunits and the formation of regulated MCU complexes [47]. However, if EMRE proteolysis is impaired in the absence of the $m$-AAA protease, excess EMRE can bind to MCU independently of MICU regulatory proteins resulting in the accumulation of constitutively open MCU-EMRE complexes (Figure 2) [47, 50]. These complexes also accumulate upon expression of an EMRE variant that is resistant to proteolysis by the $m$-AAA protease [50], substantiating the critical role of EMRE degradation for MCU complex function. Of note, the relative expression of MCU, EMRE and MICU regulatory proteins in a cell will determine how impaired proteolysis of EMRE affects mitochondrial $\mathrm{Ca}^{2+}$ influx and cell survival, providing a rationale for the cell-type specificity in disease [64].

\section{Does deregulated MCU assembly cause neurode- generation in SCA28?}

With AFG3L2 being responsible for the safety mechanism of degradation of unassembled EMRE, it is easy to envision that the accumulation of MCU-EMRE complexes and a massive mitochondrial $\mathrm{Ca}^{2+}$ influx will trigger $\mathrm{Ca}^{2+}$-induced death of $m$-AAA protease-deficient neurons [47]. Consistently, neuronal mitochondria lacking the $m$-AAA protease show an increased sensitivity to mitochondrial $\mathrm{Ca}^{2+}$ overload and opening of the MPTP [47]. This suggests that an excitotoxic increase of the mitochondrial $\mathrm{Ca}^{2+}$ concentration may induce Purkinje cell death in SCA28. The deleterious effect of the formation of ungated MCU complexes and massive mitochondrial $\mathrm{Ca}^{2+}$ influx on the survival of Purkinje cells is demonstrated by the ataxic phenotype of Micul-deficient mice [62]. Notably, such a scenario may also explain why limiting the cytosolic $\mathrm{Ca}^{2+}$ accumulation suppresses ataxia in $\mathrm{Afg} 3 \mathrm{l} 2^{+/}$mice [25] and may open up new possibilities for therapeutic interventions using specific MCU inhibitors [65].

To examine the relevance of mitochondrial $\mathrm{Ca}^{2+}$ overload for neuronal cell death, we recently deleted simultaneously $\mathrm{Mcu}$ and $\mathrm{Afg} 3 \mathrm{l2}$ specifically in mouse Purkinje cells. To this purpose we crossed $\mathrm{Mcu}^{A t / f}$ (EMMA ID07445) [66, 67] or Afg3l2 ${ }^{A / f l}$ [27] mice with transgenic mice expressing Cre recombinase under the control of the L7 promoter, which drives the expression of the transgene between 2 and 3 weeks of age, i.e. when neurogenesis is completed [68]. The genetic background was shown to play an important role in the MCU phenotype with C57BL/6 whole-body knockout mice being lethal. In contrast, $\mathrm{C} 57 \mathrm{BL} / 6$ mice lacking MCU specifically

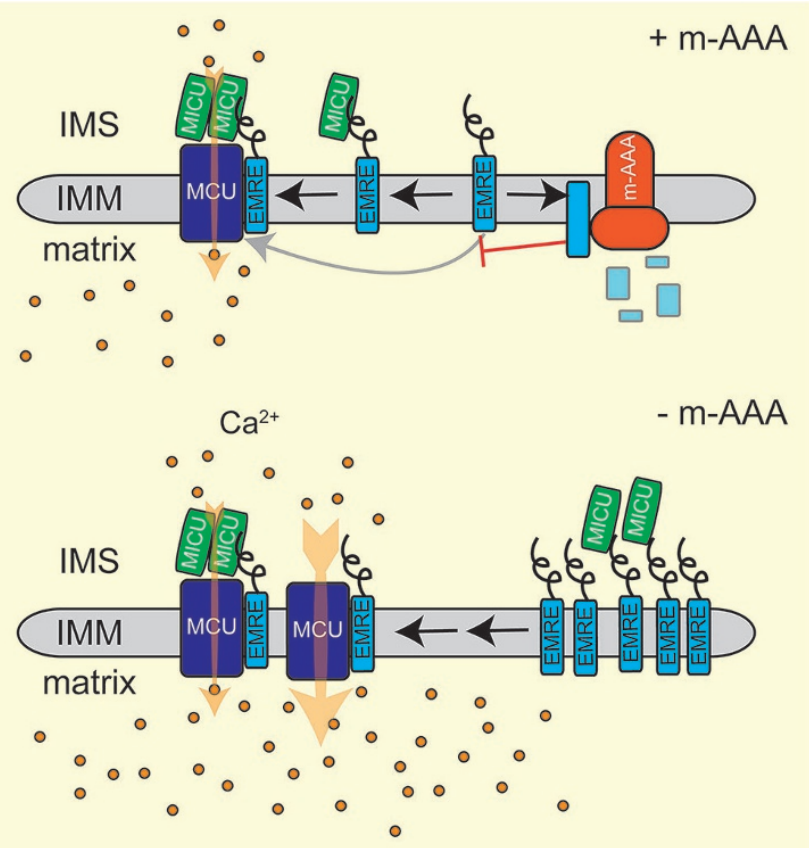

Figure 2 The $m$-AAA protease regulates MCU complex assembly by mediating the degradation of non-assembled EMRE subunits. An EMRE-MICU assembly intermediate binds to MCU subunits to form a gated MCU complex, while the m-AAA protease degrades non-assembled EMRE subunits. In the absence of the $m$-AAA protease, excess EMRE can bind either to MICU to form gated MCU complexes or can interact directly with MCU resulting in the formation of constitutively open MCU complexes lacking MICU regulatory proteins. This increases the vulnerability of cells to $\mathrm{Ca}^{2+}$ overload and $\mathrm{Ca}^{2+}$-induced cell death.

in Purkinje cells were born at the expected Mendelian ratio and did not exhibit any apparent phenotype. We observed a significant reduction of MCU protein level and $\mathrm{Ca}^{2+}$ uptake in isolated cerebellar mitochondria (data not shown). As MCU is considered to be the only pathway for the rapid uptake of $\mathrm{Ca}^{2+}$ into mitochondria, mitochondrial $\mathrm{Ca}^{2+}$ buffering appears to be dispensable for the survival of Purkinje cells. In contrast, mitochondrial $\mathrm{Ca}^{2+}$ overload in the absence of the MCU regulator MICU1 triggers death of Purkinje cells [62]. Notably, these findings also exclude impaired mitochondrial $\mathrm{Ca}^{2+}$ uptake in AFG3L2-deficient neuronal mitochondria as the pathogenic mechanism leading to neuronal loss in SCA28 [25]. On the other hand, and as previously reported [27], AFG3L2-deficient Purkinje cells degenerate starting from 6 weeks of age (Figure 3 ) and this is accompanied by an altered arborization and neuroinflammation (Figure 3). Strikingly, deletion of $\mathrm{Mcu}$ in Purkinje cells lacking AFG3L2 does not suppress neurodegeneration or the neuroinflammatory response, demonstrating that inhibiting 

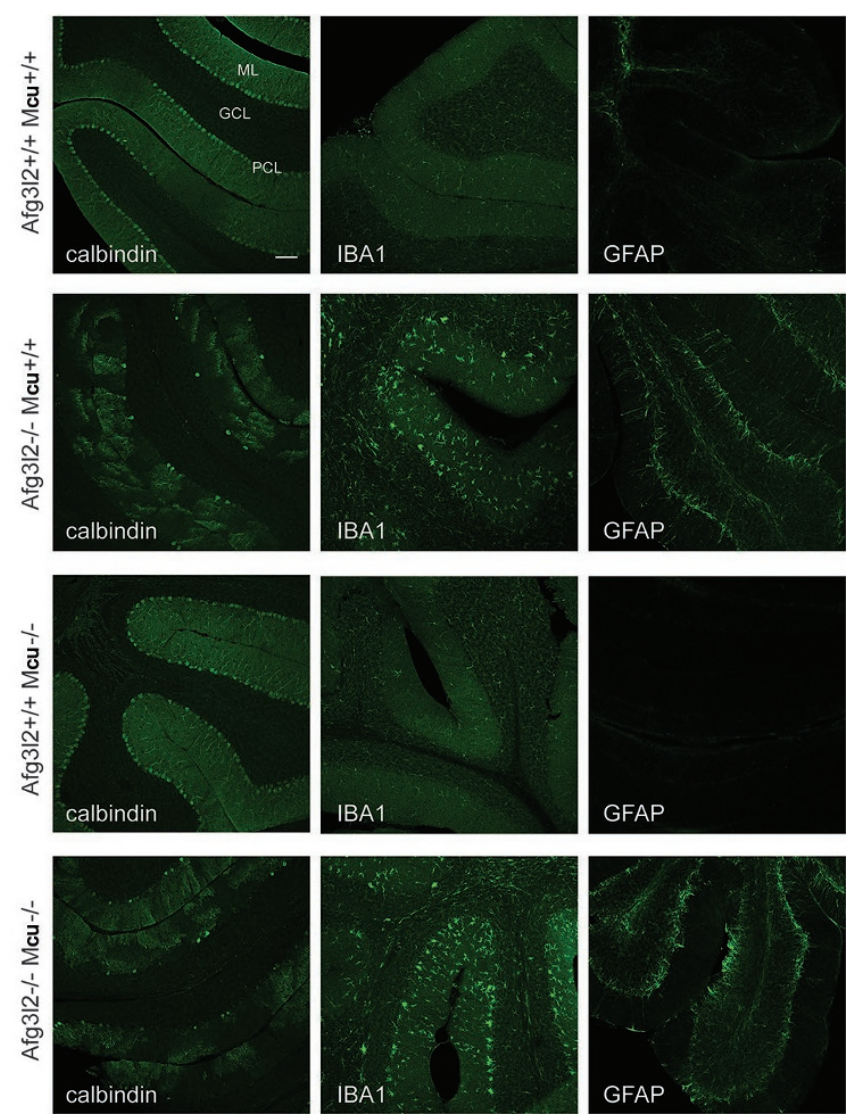

Figure 3 The deletion of $M c u$ does not prevent the degeneration of Purkinje cells lacking AFG3L2. Afg $3 / 2^{f / / f l}$ and $M c u^{f / f / l}$ were bred with transgenic mice-expressing Cre recombinase under the control of the L7 promoter specifically in Purkinje cells [68]. Cerebelli of 6-week-old offsprings of the indicated phenotypes were stained with calbindin to visualize cerebellar Purkinje cells, IBA1 to mark activated microglia and GFAP for reactive astrocytes (as described in ref. [27]). The experiments are in agreement with the national ethical guidelines for studies in animals (8402.04.2015.A402). GCL, granule cells layer; PCL, Purkinje cell layer; $\mathrm{ML}$, molecular cell layer. A representative picture is shown for each condition. Scale bar, $100 \mu \mathrm{m}$.

mitochondrial $\mathrm{Ca}^{2+}$ uptake does not preserve the survival of Purkinje cells in this model (Figure 3). These results challenge the view that disturbed mitochondrial $\mathrm{Ca}^{2+}$ homeostasis and mitochondrial $\mathrm{Ca}^{2+}$ overload is solely responsible for neuronal death in SCA28. In view of the multiple functions of the $m$-AAA protease in mitochondria, it is conceivable that an impaired mitochondrial $\mathrm{Ca}^{2+}$ homeostasis in concert with other mitochondrial deficiencies triggers the loss of Purkinje cells in SCA28.

\section{Future perspectives}

Recent years have seen major advances in our under- standing of the role of $m$-AAA proteases in mitochondria and the pathogenesis of related neurodegenerative disorders. However, the pathogenic mechanisms linking mitochondrial defects in the absence of $m$-AAA proteases with neuronal loss and clinical manifestations of the diseases are still poorly understood. The identification of the MCU subunit EMRE as a substrate of the m-AAA protease, the regulatory role of EMRE proteolysis for MCU assembly and not least the protective effect of reduced cytosolic $\mathrm{Ca}^{2+}$ levels against ataxia in $\mathrm{Afg} 3 \mathrm{ll} 2^{+/-}$mice all link disturbed mitochondrial $\mathrm{Ca}^{2+}$ homeostasis to SCA28. However, it is becoming evident that the pleiotropic functions of $m$-AAA proteases in mitochondria must be considered when analyzing disease pathogenesis. Future progress will therefore critically depend on the identification of further substrate proteins of AAA proteases. The characterization of the neuronal interactome of AFG3L2 may help in this way. These studies may also reveal whether functional differences between homo- and hetero-oligomeric $m$-AAA proteases exist and whether the striking cell-type specificity in HSP7 and SCA28 can be solely explained by tissue-specific differences in the expression of SPG7 and AFG3L2.

\section{Acknowledgments}

We thank Drs Astrid Schauss and Christian Jüngst (CECAD imaging facility) for technical support. This work was supported by EMBO fellowship to MP (ALTF 649-2015; LTFCOFUND2013; and GA-2013-609409) and grants of the Deutsche Forschungsgemeinschaft to TL.

\section{References}

1 Bohovych I, Chan SS, Khalimonchuk O. Mitochondrial protein quality control: the mechanisms guarding mitochondrial health. Antioxid Redox Signal 2015; 22:977-994.

2 Quiros PM, Langer T, Lopez-Otin C. New roles for mitochondrial proteases in health, ageing and disease. Nat Rev Mol Cell Biol 2015; 16:345-359.

3 Atorino L, Silvestri L, Koppen M, et al. Loss of m-AAA protease in mitochondria causes complex I deficiency and increased sensitivity to oxidative stress in hereditary spastic paraplegia. J Cell Biol 2003; 163:777-787.

4 Koppen M, Metodiev MD, Casari G, Rugarli EI, Langer T. Variable and tissue-specific subunit composition of mitochondrial m-AAA protease complexes linked to hereditary spastic paraplegia. Mol Cell Biol 2007; 27:758-767.

5 Gerdes F, Tatsuta T, Langer T. Mitochondrial AAA proteases - towards a molecular understanding of membrane-bound proteolytic machines. Biochim Biophys Acta 2012; 1823:49-55.

6 Kremmidiotis G, Gardner AE, Settasatian C, Savoia A, Sutherland GR, Callen DF. Molecular and functional analyses of the human and mouse genes encoding AFG3L1, a mitochondrial metalloprotease homologous to the human spastic paraplegia protein. Genomics 2001; 76:58-65. 
7 Lee S, Augustin S, Tatsuta T, Gerdes F, Langer T, Tsai FT. Electron cryomicroscopy structure of a membrane-anchored mitochondrial AAA protease. J Biol Chem 2011; 286:44044411.

8 Leonhard K, Stiegler A, Neupert W, Langer T. Chaperone-like activity of the AAA domain of the yeast Yme1 AAA protease. Nature 1999; 398:348-351.

9 Leonhard K, Guiard B, Pellecchia G, Tzagoloff A, Neupert W, Langer T. Membrane protein degradation by AAA proteases in mitochondria: extraction of substrates from either membrane surface. Mol Cell 2000; 5:629-638.

10 Graef M, Seewald G, Langer T. Substrate recognition by AAA(+) ATPases: distinct substrate binding modes in ATP-dependent protease Ymel of the mitochondrial intermembrane space. Mol Cell Biol 2007; 27:2476-2485.

11 Tatsuta T, Langer T. Quality control of mitochondria: protection against neurodegeneration and ageing. EMBO J 2008; 27:306-314.

12 Augustin S, Gerdes F, Lee S, Tsai FT, Langer T, Tatsuta T. An intersubunit signaling network coordinates ATP hydrolysis by m-AAA proteases. Mol Cell 2009; 35:574-585.

13 Tatsuta T, Augustin S, Nolden M, Friedrichs B, Langer T. $\mathrm{m}$-AAA protease-driven membrane dislocation allows intramembrane cleavage by rhomboid in mitochondria. EMBO J 2007; 26:325-335.

14 Bonn F, Tatsuta T, Petrungaro C, Riemer J, Langer T. Presequence-dependent folding ensures MrpL32 processing by the m-AAA protease in mitochondria. EMBO J 2011; 30:25452556.

15 Hartmann B, Wai T, Hu H, et al. Homozygous YME1L1 mutation causes mitochondriopathy with optic atrophy and mitochondrial network fragmentation. Elife 2016; 5:pii: e16078.

16 Casari G, De Fusco M, Ciarmatori S, et al. Spastic paraplegia and OXPHOS impairment caused by mutations in paraplegin, a nuclear-encoded mitochondrial metalloprotease. Cell 1998; 93:973-983.

17 Di Bella D, Lazzaro F, Brusco A, et al. Mutations in the mitochondrial protease gene AFG3L2 cause dominant hereditary ataxia SCA28. Nat Genet 2010; 42:313-321.

18 Cagnoli C, Mariotti C, Taroni F, et al. SCA28, a novel form of autosomal dominant cerebellar ataxia on chromosome 18p11.22-q11.2. Brain 2006; 129:235-242.

19 Pierson TM, Adams D, Bonn F, et al. Whole-exome sequencing identifies homozygous AFG3L2 mutations in a spastic ataxia-neuropathy syndrome linked to mitochondrial m-AAA proteases. PLoS Genet 2011; 7:e1002325.

20 Martinelli P, La Mattina V, Bernacchia A, et al. Genetic interaction between the m-AAA protease isoenzymes reveals novel roles in cerebellar degeneration. Hum Mol Genet 2009; 18:2001-2013.

21 Ferreirinha F, Quattrini A, Pirozzi M, et al. Axonal degeneration in paraplegin-deficient mice is associated with abnormal mitochondria and impairment of axonal transport. J Clin Invest 2004; 113:231-242.

22 Maltecca F, Aghaie A, Schroeder DG, et al. The mitochondrial protease AFG3L2 is essential for axonal development. $J$ Neurosci 2008; 28:2827-2836.

23 Wang SY, Jacquemyn J, Murru S, et al. The mitochondrial m-AAA protease prevents demyelination and hair greying.
PLoS Genet 2016; 12:e1006463.

24 Maltecca F, Magnoni R, Cerri F, Cox GA, Quattrini A, Casari G. Haploinsufficiency of AFG3L2, the gene responsible for spinocerebellar ataxia type 28 , causes mitochondria-mediated purkinje cell dark degeneration. J Neurosci 2009; 29:92449254.

25 Maltecca F, Baseggio E, Consolato F, et al. Purkinje neuron $\mathrm{Ca}^{2+}$ influx reduction rescues ataxia in SCA28 model. J Clin Invest 2015; 125:263-274.

26 Shakkottai VG, Chou CH, Oddo S, et al. Enhanced neuronal excitability in the absence of neurodegeneration induces cerebellar ataxia. J Clin Invest 2004; 113:582-590.

27 Almajan ER, Richter R, Paeger L, et al. AFG3L2 supports mitochondrial protein synthesis and Purkinje cell survival. $J$ Clin Invest 2012; 122:4048-4058.

28 Kondadi AK, Wang S, Montagner S, et al. Loss of the m-AAA protease subunit AFG3L2 causes mitochondrial transport defects and tau hyperphosphorylation. EMBO J 2014; 33:10111026.

29 Ehses S, Raschke I, Mancuso G, et al. Regulation of OPA1 processing and mitochondrial fusion by m-AAA protease isoenzymes and OMA1. J Cell Biol 2009; 187:1023-1036.

30 Head B, Griparic L, Amiri M, Gandre-Babbe S, van der Bliek AM. Inducible proteolytic inactivation of OPA1 mediated by the OMA1 protease in mammalian cells. J Cell Biol 2009; 187:959-966.

31 Anand R, Wai T, Baker MJ, et al. The i-AAA protease YME1L and OMA1 cleave OPA1 to balance mitochondrial fusion and fission. J Cell Biol 2014; 204:919-929.

32 MacVicar T, Langer T. OPA1 processing in cell death and disease - the long and short of it. J Cell Sci 2016; 129:22972306.

33 Hornig-Do HT, Tatsuta T, Buckermann A, et al. Nonsense mutations in the COX1 subunit impair the stability of respiratory chain complexes rather than their assembly. EMBO J 2012; 31:1293-1307.

34 Richter U, Lahtinen T, Marttinen P, Suomi F, Battersby BJ. Quality control of mitochondrial protein synthesis is required for membrane integrity and cell fitness. J Cell Biol 2015; 211:373-389.

35 Nolden M, Ehses S, Koppen M, Bernacchia A, Rugarli EI, Langer T. The m-AAA protease defective in hereditary spastic paraplegia controls ribosome assembly in mitochondria. Cell 2005; 123:277-289.

36 Kasumu A, Bezprozvanny I. Deranged calcium signaling in Purkinje cells and pathogenesis in spinocerebellar ataxia 2 (SCA2) and other ataxias. Cerebellum 2012; 11:630-639.

37 Schöls L, Bauer P, Schmidt T, Schulte T, Riess O. Autosomal dominant cerebellar ataxias: clinical features, genetics, and pathogenesis. Lancet Neurol 2004; 3:291-304.

38 Szabadkai G, Simoni AM, Rizzuto R. Mitochondrial $\mathrm{Ca}^{2+}$ uptake requires sustained $\mathrm{Ca}^{2+}$ release from the endoplasmic reticulum. J Biol Chem 2003; 278:15153-15161.

39 Glitsch MD, Bakowski D, Parekh AB. Store-operated $\mathrm{Ca}^{2+}$ entry depends on mitochondrial $\mathrm{Ca}^{2+}$ uptake. EMBO J 2002; 21:6744-6754.

40 Rizzuto R, De Stefani D, Raffaello A, Mammucari C. Mitochondria as sensors and regulators of calcium signalling. Nat Rev Mol Cell Biol 2012; 13:566-578. 
41 Bernardi P, Rasola A, Forte M, Lippe G. The mitochondrial permeability transition pore: channel formation by F-Atp synthase, integration in signal transduction, and role in pathophysiology. Physiol Rev 2015; 95:1111-1155.

42 Bernardi P, Scorrano L, Colonna R, Petronilli V, Di Lisa F. Mitochondria and cell death - mechanistic aspects and methodological issues. Eur J Biochem 1999; 264:687-701.

43 Santos JB, Schauwecker PE. Protection provided by cyclosporin A against excitotoxic neuronal death is genotype dependent. Epilepsia 2003; 44:995-1002.

44 Shanmughapriya S, Rajan S, Hoffman NE, et al. SPG7 is an essential and conserved component of the mitochondrial permeability transition pore. Mol Cell 2015; 60:47-62.

45 Lee SG, Su ZZ, Emdad L, et al. Mechanism of ceftriaxone induction of excitatory amino acid transporter-2 expression and glutamate uptake in primary human astrocytes. $\mathrm{J}$ Biol Chem 2008; 283:13116-13123.

46 Maltecca F, De Stefani D, Cassina L, et al. Respiratory dysfunction by AFG3L2 deficiency causes decreased mitochondrial calcium uptake via organellar network fragmentation. Hum Mol Genet 2012; 21:3858-3870.

47 König T, Tröder SE, Bakka K, et al. The m-AAA protease associated with neurodegeneration limits MCU activity in mitochondria. Mol Cell 2016; 64:148-162.

48 Clements A, Bursac D, Gatsos X, et al. The reducible complexity of a mitochondrial molecular machine. Proc Natl Acad Sci USA 2009; 106:15791-15795.

49 Sancak Y, Markhard AL, Kitami T, et al. EMRE is an essential component of the mitochondrial calcium uniporter complex. Science 2013; 342:1379-82.

50 Tsai CW, Wu Y, Pao PC, et al. Proteolytic control of the mitochondrial calcium uniporter complex. Proc Natl Acad Sci USA 2017; 114:4388-4393.

51 De Stefani D, Raffaello A, Teardo E, Szabò I, Rizzuto R. A forty-kilodalton protein of the inner membrane is the mitochondrial calcium uniporter. Nature 2011; 476:336-340.

52 Baughman JM, Perocchi F, Girgis HS, et al. Integrative genomics identifies MCU as an essential component of the mitochondrial calcium uniporter. Nature 2011; 476:341-345.

53 Mammucari C, Gherardi G, Rizzuto R. Structure, activity regulation, and role of the mitochondrial calcium uniporter in health and disease. Front Oncol 2017; 7:139.

54 Raffaello A, De Stefani D, Sabbadin D, et al. The mitochondrial calcium uniporter is a multimer that can include a dominant-negative pore-forming subunit. EMBO J 2013; 32:23622376.

55 Plovanich M, Bogorad RL, Sancak Y, et al. MICU2, a paralog of MICU1, resides within the mitochondrial uniporter complex to regulate calcium handling. PLoS One 2013; 8:e55785.

56 Patron M, Checchetto V, Raffaello A, et al. MICU1 and MICU2 finely tune the mitochondrial $\mathrm{Ca}^{2+}$ uniporter by exerting opposite effects on MCU activity. Mol Cell 2014; 53:726737.

57 Perocchi F, Gohil VM, Girgis HS, et al. MICU1 encodes a mitochondrial $\mathrm{EF}$ hand protein required for $\mathrm{Ca}^{2+}$ uptake. $\mathrm{Na}$ ture 2010; 467:291-296.

58 Csordás G, Golenár T, Seifert EL, et al. MICU1 controls both the threshold and cooperative activation of the mitochondrial $\mathrm{Ca}^{2+}$ uniporter. Cell Metab 2013; 17:976-987.
59 Mallilankaraman K, Doonan P, Cárdenas C, et al. MICU1 is an essential gatekeeper for MCU-mediated mitochondrial $\mathrm{Ca}^{2+}$ uptake that regulates cell survival. Cell 2012; 151:630644.

60 Kovács-Bogdán E, Sancak Y, Kamer KJ, et al. Reconstitution of the mitochondrial calcium uniporter in yeast. Proc Natl Acad Sci USA 2014; 111:8985-8990.

61 Tsai MF, Phillips CB, Ranaghan M, et al. Dual functions of a small regulatory subunit in the mitochondrial calcium uniporter complex. Elife 2016; 5:pii: e15545.

62 Liu JC, Liu J, Holmström KM, et al. MICU1 serves as a molecular gatekeeper to prevent in vivo mitochondrial calcium overload. Cell Rep 2016; 16:1561-1573.

63 Logan CV, Szabadkai G, Sharpe JA, et al. Loss-of-function mutations in MICU1 cause a brain and muscle disorder linked to primary alterations in mitochondrial calcium signaling. Nat Genet 2014; 46:188-193.

64 Paillard M, Csordás G, Szanda G, et al. Tissue-specific mitochondrial decoding of cytoplasmic $\mathrm{Ca}^{2+}$ signals is controlled by the stoichiometry of MICU1/2 and MCU. Cell Rep 2017; 18:2291-2300.

65 Arduino DM, Wettmarshausen J, Vais H, et al. Systematic identification of MCU modulators by orthogonal interspecies chemical screening. Mol Cell 2017; 67:711-723.e.7.

66 Bradley A, Anastassiadis K, Ayadi A, et al. The mammalian gene function resource: the international knockout mouse consortium. Mamm Genome 2012; 23:580-586.

67 Meehan TF, Conte N, West DB, et al. Disease model discovery from 3,328 gene knockouts by The International Mouse Phenotyping Consortium. Nat Genet 2017; 49:1231-1238.

68 Barski JJ, Dethleffsen K, Meyer M. Cre recombinase expression in cerebellar Purkinje cells. Genesis 2000; 28:93-98.

69 Jenkinson EM, Rehman AU, Walsh T, et al. Perrault syndrome is caused by recessive mutations in CLPP, encoding a mitochondrial ATP-dependent chambered protease. Am J Hum Genet 2013; 92:605-613.

70 Johnson F, Kaplitt MG. Novel mitochondrial substrates of Omi indicate a new regulatory role in neurodegenerative disorders. PLoS One 2009; 4:e7100.

71 Clausen T, Kaiser M, Huber R, Ehrmann M. HTRA proteases: regulated proteolysis in protein quality control. Nat Rev Mol Cell Biol 2011; 12:152-162.

72 Simon-Sanchez J, Singleton AB. Sequencing analysis of OMI/HTRA2 shows previously reported pathogenic mutations in neurologically normal controls. Hum Molec Genet 2008; 17:1988-1993.

73 Bertelsen B, Melchior L, Jensen LR, et al. Intragenic deletions affecting two alternative transcripts of the IMMP2L gene in patients with Tourette syndrome. Eur J Hum Genet 2014; 22:1283-1289.

74 Strauss KA, Jinks RN, Puffenberger EG, et al. CODAS syndrome is associated with mutations of LONP1, encoding mitochondrial AAA(+) lon protease. Am J Hum Genet 2015; 96:121-135.

75 Shi G, Lee JR, Grimes DA, et al. Functional alteration of PARL contributes to mitochondrial dysregulation in Parkinson's disease. Hum Mol Genet 2011; 20:1966-1974.

76 Fields S, Song OK. A novel genetic system to detect protein protein interactions. Nature 1989; 340:245-246. 
77 Koutnikova H, Campuzano V, Koenig M. Maturation of wildtype and mutated frataxin by the mitochondrial processing peptidase. Hum Mol Genet 1998; 7:1485-1489.

78 O'Toole JF, Liu Y, Davis EE, et al. Individuals with mutations in XPNPEP3, which encodes a mitochondrial protein, develop a nephronophthisis-like nephropathy. J Clin Invest 2010; 120:791-802.

79 Vig PJ, Subramony SH, McDaniel DO. Calcium homeostasis and spinocerebellar ataxia-1 (SCA-1). Brain Res Bull 2001; 56:221-225.

80 Matilla-Duenas A, Goold R, Giunti P. Clinical, genetic, molecular, and pathophysiological insights into spinocerebellar ataxia type 1. Cerebellum 2008; 7:106-114.

81 Lastres-Becker I, Rub U, Auburger G. Spinocerebellar ataxia 2 (SCA2). Cerebellum 2008; 7:115-124.

82 Kasumu A, Bezprozvanny I. Deranged calcium signaling in purkinje cells and pathogenesis in spinocerebellar ataxia 2 (SCA2) and other ataxias. Cerebellum 2012; 11:630-639.

83 Riess O, Rüb U, Pastore A, Bauer P, Schöls L. SCA3: Neurological features, pathogenesis and animal models. Cerebellum 2008; 7:125-137.

84 Ikeda Y, Ikeda Y, Day JW, Ranum LP. Spectrin mutations cause spinocerebellar ataxia type 5. Nat Genet 2006; 38:184190.
85 Zhuchenko O, Bailey J, Bonnen P, et al. Autosomal dominant cerebellar ataxia (SCA6) associated with polyglutamine expansion in the alpha(1A)-voltage-dependent calcium channel. Nat Genet 1997; 15:62-69.

86 Chen DH, Brkanac Z, Verlinde CL, et al. Missense mutations in the regulatory domain of PKC gamma: a new mechanism for dominant nonepisodic cerebellar ataxia. Am J Hum Genet 2003; 72:839-849.

87 Knight MA, Kennerson ML, Anney RJ, et al. Spinocerebellar ataxia type 15 (SCA15) maps to 3p24.2-3pter: exclusion of the ITPR1 gene, the human orthologue of an ataxic mouse mutant. Neurobiol Dis 2003; 13:147-157.

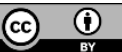

This work is licensed under a Creative Commons Attribution 4.0 Unported License. The images or other third party material in this article are included in the article's Creative Commons license, unless indicated otherwise in the credit line; if the material is not included under the Creative Commons license, users will need to obtain permission from the license holder to reproduce the material. To view a copy of this license, visit http:// creativecommons.org/licenses/by/4.0/

(C) The Author(s) 2018 